\title{
Incidence and Clinical Pattern of Proximal Humeral Fractures of Patients Fixed by Locking Proximal Humeral Plate (LPHP)
}

\author{
Dr. A. K. M. Harun-Ar-Rashid ${ }^{1 *}$, Dr. Abdur Rahman ${ }^{2}$, Dr. Mohammad Shaha Alam³ ${ }^{3}$ Dr. Ayesha Begum ${ }^{4}$, Dr. A. H. M.
} Azgar Ali Chowdhury ${ }^{5}$, Dr. A.T. M. Rezaul Karim ${ }^{6}$

\footnotetext{
${ }^{1}$ Assistant Professor of Orthopaedic Surgery, Cox's Bazar Medical College, Cox’s Bazar, Bangladesh

${ }^{2}$ Assistant Professor of Orthopaedic Surgery, Chattogram Medical College, Chattogram, Bangladesh

${ }^{3}$ Assistant. Professor of Surgery, Cox’s Bazar Medical College, Cox's Bazar, Bangladesh

${ }^{4}$ Assistant Professor of Paediatrics, Chattogram Medical College, Chattogram, Bangladesh

${ }^{5}$ Junior Consultant of Orthopaedic Surgery, Chittagong Medical College Hospital, Chattogram, Bangladesh

${ }^{6}$ Consultant of Orthopaedic Surgery, Parkview Hospital, Chattogram, Bangladesh
}

DOI: $10.36347 /$ sjams.2021.v09i04.022

| Received: 03.03.2021 | Accepted: 22.03.2021 | Published: 24.04.2021

*Corresponding author: Dr. A. K. M. Harun-Ar-Rashid

Abstract

Original Research Article

Objective: In this study our main goal is to evaluate the functional outcome of the treatment of proximal humeral fractures fixed by Locking Proximal Humeral Plate (LPHP). Method: This prospective observational study was carried out at Department of Orthopaedic Surgery, Chittagong Medical College Hospital, Chittagong. All the 30 patients are above 18 years of age and was admitted in different units of Orthopaedic ward of CMCH with displaced fractures of the proximal humerus of both sexes. Results: During the study, of the 30 patients, $16(53.33 \%)$ presented with right sided proximal humeral fracture and 14(46.66\%) with left sided fractures. Also, of the 30 patients, 11(36.66\%) 2 part, $13(43.33 \%)$ presented with three-part fractures. 1(3.33\%) with four-part fractures and 5(16.66\%) with fracture dislocations. According to Constant score criteria 20(66.7\%) patients can externally rotate their shoulder with hand above head and elbow forward, $6(20 \%)$ patients can do it with hand behind head and elbow backward, 2(6.7\%) each can do it either with hand behind head and elbow forward or hand above head and elbow backward. Conclusion: From our study we can conclude that, the results show that in proximal humeral fractures treated by open reduction and internal fixation with locking proximal humeral plate (LPHP) is the good method of treatment in the absence of C-arm image intensifier.

Keywords: proximal humeral fractures, locking proximal humeral plate (LPHP), plate fixation.

Copyright $(\odot 2021$ The Author(s): This is an open-access article distributed under the terms of the Creative Commons Attribution 4.0 International License (CC BY-NC 4.0) which permits unrestricted use, distribution, and reproduction in any medium for non-commercial use provided the original author and source are credited.

\section{INTRODUCTION}

Fractures of the proximal humerus have challenged medical practitioners since the beginning of recorded medical history. In the earliest known surgical text, the Edwin smith papyrus (1600 BC), three cases of humeral fractures were described [1]. Hippocrates is credited with documenting the first fracture of the proximal humerus in $460 \mathrm{BC}$ and describing a method of weight traction that aided in bone healing. The Alexandrian school of medicine (third century BC) mentioned shoulder dislocations complicated with fractures and the author discussed whether the dislocation should be reduced before or after the fracture [2]. Celsus (25 BC-AD 50) distinguished shaft fractures from the proximal and distal humeral fractures. Several illustrations from the sixteenth and seventeenth century surgical texts showed the ancient methods of reduction and bandaging. Long term results of orthopedic treatments were not systematically recorded until the late eighteenth century when pathoanatomic findings from autopsy began to be compared with clinical observations by renown pathologist like astley Cooper [3].

An anatomical classification was developed in the late $19^{\text {th }}$ century in an attempt to improve the diagnosis and treatment but this simplified scheme was not thorough enough and lacked consistency. A significant contribution was made by Codman when he divided proximal humerus into four parts. These parts were divided along epiphyseal lines and consisted of the head, greater tuberosity, lesser tuberosity and shaft [4].

Neer's classification was the most comprehensive and widely accepted one to classify 
A. K. M. Harun-Ar-Rashid et al; Sch J App Med Sci, Apr, 2021; 9(4): 597-600

proximal humeral fractueres with integration of fracture anatomy, biomechanics, and displacement [5].

In this study our main goal is to evaluate the functional outcome of the treatment of proximal humeral fractures fixed by Locking Proximal Humeral Plate (LPHP).

\section{OBJECTIVE}

To evaluate the functional outcome of the treatment of proximal humeral fractures fixed by Locking Proximal Humeral Plate (LPHP).

\section{METHODOLOGY}

Study design: This was a prospective observational study.

Place of study: This study was carried out at Department of Orthopaedic Surgery, Chittagong Medical College Hospital, Chittagong.

Study population: All the patients are above 18 years of age and was admitted in different units of Orthopaedic ward of $\mathrm{CMCH}$ with displaced fractures of the proximal humerus of both sexes. For diagnosis Neer's classification of the proximal humerus fracture was used. Cases were selected purposively.

Sample size: Total 34 patients were enrolled in the study. Four patients were lost to follow-up. So, finally 30 patients were available for evaluation.

\section{Inclusion Criteria}

Patients with following characteristics will be included in the study.

- Patients with closed displaced two, three- or fourpart fractures and fracture dislocations.

- Age above 18 years.

- The patients who are mentally fit and physically alert (ASA-grorp 1-3)

- Patients without any neurovascular disorder.

\section{Exclusion Criteria}

Patients with following characteristics will be excluded from the study.

- Open fracture

- Pathological fracture

- Previous shoulder surgery

- Chronic shoulder pain

- Ipsilateral upper limb fractures

\section{Data Collection}

Pretested and predesigned pro-forma containing history and examination finding of the patients and operative procedure \& follow up were used to collect the data as was approved in the protocol.

\section{Data analysis}

Once data collection was completed, data was compiled and tabulated according to key variables.
Analysis of different variables were done according to standard statistical method and calculations were done using scientific calculators \& using MS-excel program in computer.

\section{RESULTS}

Table-1 demonstrates age distribution where out of 30 patients $5(16.66 \%)$ were 26 to 35 years of age, $3(10 \%)$ were 36 to 45 years old, $5(16.66 \%)$ were 46 to 55 years old, $15(50 \%)$ were 56 to 65 years, and $2(6.66 \%)$ patient were 66 to 75 years old. The mean age of the patients was 63.13 years and the youngest and the oldest patient was 26 years and 75 years respectively. The following table is given below in detail:

Table-1: Age distribution of the patients $(n=30)$

\begin{tabular}{|l|l|l|}
\hline Age in years & Frequency & Percentage \\
\hline $26-35$ & 5 & 16.66 \\
\hline $36-45$ & 3 & 10 \\
\hline $46-55$ & 5 & 16.66 \\
\hline $56-65$ & 15 & 50 \\
\hline $66-75$ & 2 & 6.66 \\
\hline
\end{tabular}

Mean age $=63.13$ years, Range-26 to 75 yrs.

In Figure-1 shows sex distribution. Majority $(56.66 \%)$ of the patients were female and the rest $(43.33 \%)$ male giving a male to female ratio of roughly $1.30: 1$. The following figure is given below in detail:

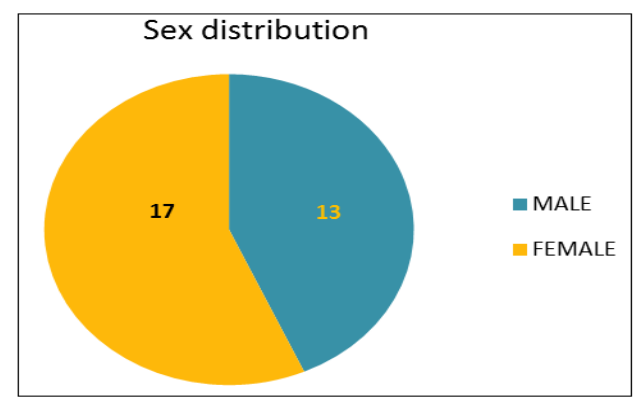

Fig-1: Pie chart shows Sex distribution of the patients $(n=30)$

In Figure-2 shows affected limb of the patients where of the 30 patients, $16(53.33 \%)$ presented with right sided proximal humeral fracture and 14(46.66\%) with left sided fractures. The following figure is given below in detail:

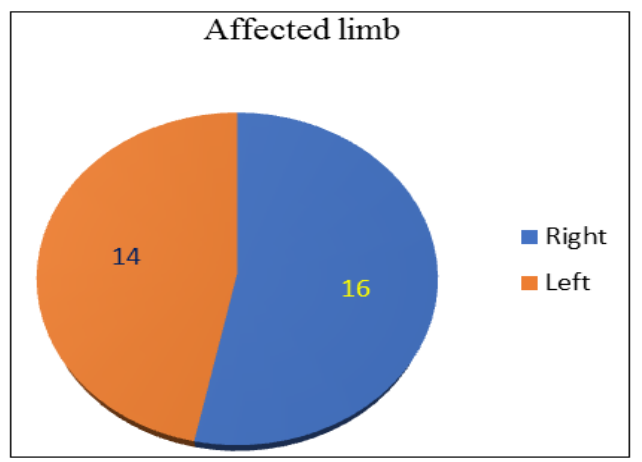

Fig-2: Pie chart shows distribution of the patients by affected limb $(\mathbf{n}=\mathbf{3 0})$ 
A. K. M. Harun-Ar-Rashid et al; Sch J App Med Sci, Apr, 2021; 9(4): 597-600

In Figure-3 shows fracture pattern of the patients where of the 30 patients, 11(36.66\%) 2 part, $13(43.33 \%)$ presented with three-part fractures. $1(3.33 \%)$ with four-part fractures and $5(16.66 \%)$ with fracture dislocations. The following figure is given below in detail:

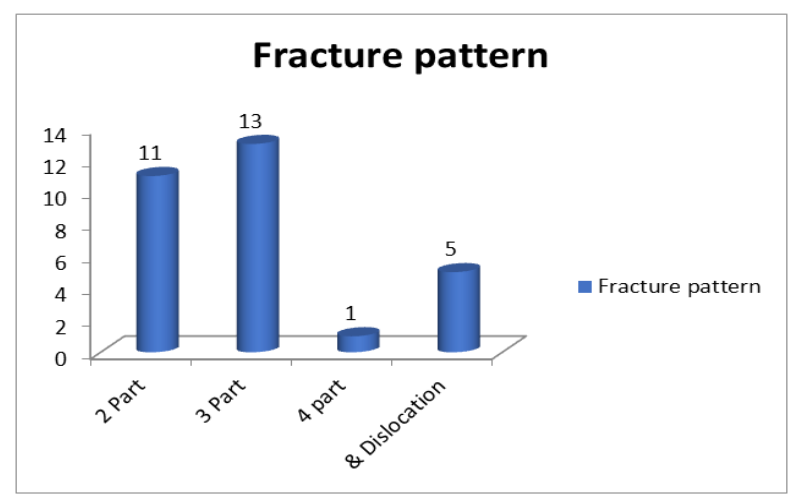

Fig-3: Bar diagram demonstrates distribution of the patients by fracture pattern $(n=30)$

In Table-2 shows Distribution of the patients by pain symptoms where at six months follows up, only $2(6.66 \%)$ patients complained of moderate pain, 12 (40\%) patients had no pain at all, $16(53.3 \%)$ patients had only mild pain. The following table is given below in detail:

Table-2: Distribution of the patients by pain symptoms $(\mathbf{n}=\mathbf{3 0})$

\begin{tabular}{|l|l|l|}
\hline Pain type & Frequency & Percentage \\
\hline No & 12 & 40 \\
\hline Mild & 16 & 53.3 \\
\hline Moderate & 2 & 6.66 \\
\hline
\end{tabular}

In Table-3 shows limitation of patients in daily living where on the final follow up at 6 to 18 months, $18(60 \%)$ patients had no limitation of daily activities,
$12(40 \%)$ patients had moderate limitation of daily activities. $16(53.3 \%)$ patients had no limitation of recreational activities and $14(46.7 \%)$ patients had moderate limitations. 20(66.7\%) patients had no sleep disturbance but $10(33.3 \%)$ patients complained of some sleep disturbance due to pain. The following table is given below in detail:

Table-3: Distribution of the patients by limitation of daily living $(\mathbf{n}=\mathbf{3 0})$

\begin{tabular}{|l|l|l|}
\hline Limitation of daily living & NO & Moderate \\
\hline Limitation of daily living & $18(60 \%)$ & $12(40 \%)$ \\
\hline $\begin{array}{l}\text { Limitation of recreational } \\
\text { activities }\end{array}$ & $16(53.3 \%)$ & $14(46.7 \%)$ \\
\hline Sleep disturbance & $20(66.7 \%)$ & $10(33.3 \%)$ \\
\hline
\end{tabular}

In Table-4 shows distribution of patients according to abduction movement. Abduction to the Constant score criteria the abduction of shoulder was $61^{0}-90^{\circ}$ in $4(13.3 \%)$ patients $91^{\circ}-120^{\circ}$ in $22(73.3 \%)$ patients and $131^{\circ}-150^{\circ}$ in $4(13.3 \%)$ patients. The following table is given below in detail:

Table-4: Distribution of patients according to abduction movement $(n=30)$

\begin{tabular}{|l|l|l|}
\hline Range of motion & Frequency & Percentage \\
\hline $61^{0}-90^{0}$ & 4 & 13.3 \\
\hline $91^{0}-120^{0}$ & 22 & 73.3 \\
\hline $121^{0}-150^{0}$ & 4 & 13.3 \\
\hline \multicolumn{2}{|c|}{$\mathbf{P}<\mathbf{0 . 0 0 1}$ in $\mathbf{x}^{\mathbf{2}}$ test, the result is highly significant }
\end{tabular}

In Table-5 shows distribution of patients according to external rotatory movement. According to Constant score criteria 20(66.7\%) patients can externally rotate their shoulder with hand above head and elbow forward, 6(20\%) patients can do it with hand behind head and elbow backward, $2(6.7 \%)$ each can do it either with hand behind head and elbow forward or hand above head and elbow backward. The following table is given below in detail:

Table-5: Distribution of patients according to external rotatory movement $(n=30)$

\begin{tabular}{|l|l|l|}
\hline Rotation & Frequency & Percentage \\
\hline Hand above head and elbow forward & 20 & 66.7 \\
\hline Hand behind head and elbow forward & 2 & 6.7 \\
\hline Hand above head and elbow backward & 2 & 6.7 \\
\hline Hand behind head and elbow backward & 6 & 20.0 \\
\hline
\end{tabular}

\section{$P<0.001$ in $x^{2}$ test, the result is highly significant}

In Table-6 shows internal rotation of the patients. According to Constant scoring criteria the dorsum of the hand reaches D12 vertebra in 4(13.3\%) patients. Waist in $18(60 \%)$ patients. Sacroiliac joint in $2(6.7 \%)$ patients. Buttock in $4(13.3 \%)$ patients and thigh in $2(6.7 \%)$ patients. The following table is given below in detail:
Table-6: Distribution of patients according to internal rotatory movement $(n=30)$

\begin{tabular}{|l|l|l|}
\hline $\begin{array}{l}\text { Dorsum of the hand } \\
\text { reaching }\end{array}$ & Frequency & Percentage \\
\hline D12 & 4 & 13.3 \\
\hline Waist & 18 & 60.0 \\
\hline SI joint & 2 & 6.7 \\
\hline Buttock & 4 & 13.3 \\
\hline Thigh & 2 & 6.7 \\
\hline
\end{tabular}

$P<0.001$ in $x^{2}$ test, the result is highly significant 
A. K. M. Harun-Ar-Rashid et al; Sch J App Med Sci, Apr, 2021; 9(4): 597-600

In Table-7 shows constant score grading. The outcome of the patients was graded according to constant score criteria as good $(>70)$ in $14(46.6 \%)$ patients, Fair (56-70) in $11(36.66 \%)$ patients and poor $(0-55)$ in $5(16.66 \%)$ patients. The following table is given below in detail:

Table-7: Distribution of patients according to Constant score grading $(\mathbf{n}=\mathbf{3 0})$

\begin{tabular}{|l|l|l|}
\hline Grading & Frequency & Percentage \\
\hline Good & 14 & 46.6 \\
\hline Fair & 11 & 36.66 \\
\hline Poor & 5 & 16.66 \\
\hline
\end{tabular}

$\mathbf{P}>0.01$ in $\mathrm{x}^{2}$ test, the result is significant.

\section{DisCUSSION}

In the present series, the right side was more affected $(60 \%)$ than the left $(40 \%)$. In one study the right side $(57 \%)$ was more affected than the left side (43\%) [6]. Whereas, in another study right and left hummers was almost equally affected [7].

The cause of left side involvement is probably due to unpreparedness following high velocity injury in most of the cases. Dominant right sides of female and older patients are more affected probably due to searching of support during low energy trauma.

In the present series, the basis of diagnosis was clinical examination and radiological evaluation of the affected part in both antero-posterior and lateral views. All the cases were displaced two, three or four part fractures and some were also associated with dislocation of the humeral head and all were treated by open reduction and internal fixation by Locking Proximal Humeral Plate (LPHP). After operation long arm back slab or U-cast was applied and kept for 2 weeks till the stitch is removal. Pendulum exercise was started as early as $2^{\text {nd }}$ week. Physiotherapy is vital for early recovery of the patient and is supervised very closely in almost all patients.

At follow ups in this series, each patient was assessed for pain, limitation of the activates of daily living and range of motion according to constantMorley shoulder assessment score 1987 [8].

At the final follow up at 6 to 18 months there was moderate pain in $6.6 \%$ patients with moderate of activity in $46.7 \%$ patients. In this series at the time of operation all the fractures were closed, during operation strict asepsis was followed in every step and broad spectrum intravenous antibiotics were given for three days. Initial recovery was uneventful in all the cases except two. In 8 patients there was superficial infection and were treated by regular local wound dressing with local and systemic antibiotic according to the culture andsensitivity report. In another 8 were persistent subluxation which needed to be corrected under C-arm guidance. Only one patient $(6.7 \%)$ did not show complete union even at 6 months. In the present series no patient developed avascular necrosis. It indicates that blood supply of the humeral head had not been critically impaired by open reduction and internal fixation by proximal humeral locking plate.

\section{CONCLUSION}

From our study we can conclude that, the results show that in proximal humeral fractures treated by open reduction and internal fixation with locking proximal humeral plate (LPHP) is the good method of treatment in the absence of $\mathrm{C}$-arm image intensifier.

\section{REFERENCE}

1. Gustilo RB, Kyle RF, Templeman DC. Fractures and Dislocations: Fractures and dislocations of the proximal humerus, scapula, Sternoclavicular joint, acromioclavicular joint and clavicle. $1^{\text {st }}$ ed. St, Louis, MO: Mosby-Yearbook, Inc 1993;1: 255340 .

2. Court-Brown CM, Garg A, Mequeen MM. The epidemiology of proximal humeral fractures. Acta Orthop Scand. 2001; 72(4):365-71.

3. Strohm PC, Ko“stler W, Su“dkamp NP. Locking plate fixation of proximal humerus fractures. Techniques in Shoulder and Elbow Surgery. 2005; 6(1):8-13.

4. Neer CS. Displaced proximal humeral fractures. classification and evaluation. J Bone Joint Surg Am. 1970; 52:1077-89.

5. Flatow EL, Cuomo F, Madey MG, miller SR, Mallueen SJ, Bigiliani LU. Open reducation and internal fixation of two part displaced fractures of greater tuberosity of the proximal part of the humerus. J bone joint surg Am. 1991; 73(8):1213 18.

6. Korkmaz MF, Aksu N, Gogus A, Debre M, Kara $\mathrm{AN}$, Isiklar $\mathrm{zv}$. the results of internal fixation of proximal humeral fractures with the philos locking plate. Acta Orthop traumatol turc. 2008; 42(2):97105.

7. Sosef N, Stobbe I, Hogervorst M, Mommers L, Verbruggen J, Elist M. the polarus intramedullary nail for proximal humerus fractures, Acta orthopaedica. 2007;78(3):436-41.

8. Robinson CM, Page RS, Hill RMF, Sanders DL, Court-Brown CM, Wakefield AE. 'Primary hemiarthroplasty for the treatment of proximal humerus fractures, J Bone Joint Surg Am. 2003;85:1215-23. 